\title{
Estado nutricional e qualidade de vida de pacientes em hemodiálise
}

\author{
Nutritional status and quality of life in hemodialysis patients
}

Mayara Ferreira Bernardo1, Eryka Maria dos Santos², Mikaella Carla de França Cavalcanti ${ }^{3}$ Denise Sandrelly Cavalcanti de Lima4

\begin{abstract}
RESUMO
Introdução: Na Doença Renal Crônica Avançada, o paciente necessita de uma terapia renal substitutiva, dentre elas, a hemodiálise (HD). Apesar dos benefícios da HD, este procedimento afeta o estado nutricional e a qualidade de vida (QV) do doente. Objetivo: Correlacionar o estado nutricional ao nível de QV de pacientes em HD. Metodologia: Estudo transversal, com pacientes em HD há pelo menos 3 meses. O estado nutricional foi avaliado pelo índice de massa corporal (IMC), circunferência do braço (CB), circunferência muscular do braço (CMB), prega cutânea tricipital (PCT), albumina (Alb) e creatinina $(\mathrm{Cr})$ séricas e a QV pelo Medical Outcomes Study-36 - Item Short Form Health Survey (SF-36). As pontuações geradas pelo SF-36 foram correlacionadas com os indicadores do estado nutricional através do Coeficiente de Correlação de Pearson e/ou Spearman's. Resultados e Discussão: Participaram do estudo 37 pacientes, com idade média de 50,6 $\pm 13,0$ anos, sendo 54,1\% do sexo feminino. Segundo a avaliação do estado nutricional, houve prevalência de eutrofia $(48,7 \%)$ pelo IMC e desnutrição de 37,8\% evidenciada pela CB e CMB. Na avaliação bioquímica, 25,0\% estavam com hipoalbuminemia e 18,9\% com redução da Cr. A dimensão de QV mais comprometida foi a limitação por aspectos físicos. Na análise das correlações, parâmetros nutricionais (IMC, CB e Alb) estavam ligados à QV dos pacientes. Conclusão: IMC, CB e Alb correlacionaram-se com dimensões do SF-36, indicando que alterações no estado nutricional podem influenciar o funcionamento físico e emocional desses pacientes.
\end{abstract}

Palavras-chave: Estado Nutricional. Qualidade de Vida. Doença Renal Crônica. Hemodiálise.

\begin{abstract}
Introduction: In Advanced Chronic Kidney Disease, the patient needs renal replacement therapy, among them, hemodialysis (HD). Despite the benefits of HD, this procedure affects the nutritional status and quality of life (QL) of the patient. Objective: Correlating the nutritional status at the QL level of patients in HD. Methods: Cross-sectional study with patients on HD for at least three months. Patients underwent an assessment of nutritional status by body mass index (BMI), arm circumference (AC),
\end{abstract}

1. Especialista em Nutrição Clínica pelo Hospital das Clínicas da Universidade Federal de Pernambuco (HC-UFPE), Recife, PE.

2. Mestranda em Cirurgia pela UFPE, Recife, PE.

3. Mestranda em Gerontologia pela UFPE, Recife, PE.

4. Doutora pela Universidade Federal de Pernambuco (UFPE), Recife, PE.
CORRESPONDÊNCIA:

Eryka Maria dos Santos Hospital das Clínicas da UFPE, Nutrição Av. Prof. Moraes Rego S/N. Cidade Universitária 50670-901- Recife/PE - Brasil erykasantos.nutri@gmail.com

Recebido em: 08/08/2018

Aprovado em: 08/05/2019 
arm muscle circumference (MAMC), triceps skinfold (TS), serum albumin (Alb) and creatinine (CR). QL was measured using the Medical Outcomes Study 36 - Item Short Form Health Survey (SF-36) questionnaire. The scores generated by the SF-36 were correlated with the nutritional status indicators through the Pearson and/or Spearman's Correlation Coefficient. Results and Discussion: 37 patients participated in the study, aged $50.6 \pm 13.0$ years old, and $54.1 \%$ were female. According to the evaluation of nutritional status, there was a prevalence of eutrophy $(48.7 \%)$ by BMI and malnutrition of $37.8 \%$ evidenced by $A C$ and MAMC. In the biochemical evaluation, $25.0 \%$ had a reduction of Alb and $18.9 \%$ of CR. The dimension of QL was the most impaired physical aspects limitation. In the analysis of correlations, nutritional parameters (BMI, AC, and Alb) were correlated to patients' QL. Conclusion: BMI, AC, and Alb correlated with SF-36 dimensions, indicating that changes in nutritional status may influence the physical and emotional functioning of these patients.

Keywords: Nutritional Status. Quality of Life. Chronic Kidney Disease. Hemodialysis.

\section{INTRODUÇÃO}

A doença renal crônica (DRC) caracteriza-se por uma lesão progressiva, gradual e irreversível da função renal, dividida em estágios baseados na taxa de filtração glomerular. Em seu estágio avançado, correspondente ao estágio 5 da classificação adotada pela Sociedade Brasileira de Nefrologia (SBN) ${ }^{1}$, geralmente há necessidade da instalação de uma terapia renal substitutiva (TRS), ou seja, realização de transplante renal ou início de terapia dialítica. A terapia dialítica compreende o uso de técnicas peritoneais ou da hemodiálise (HD), esta última é a forma de TRS mais comum utilizada no Brasil (SBN). ${ }^{2}$

Apesar de seus benefícios, a HD está fortemente ligada ao estado nutricional (EN) e a qualidade de vida (QV) do doente renal, sendo necessária a adaptação do paciente e dos familiares a um tratamento que exige intenso compromisso e dedicação, levando, consequentemente, ao cansaço e ao estresse, que interferem diretamente na QV. ${ }^{3}$

A correlação entre fatores modificáveis, como o EN, e fatores subjetivos, como o nível de $\mathrm{QV}$, pode auxiliar a equipe de saúde na abordagem desses pacientes e, também, direcionar as intervenções necessárias. ${ }^{4}$

Portanto, apesar do crescente número de pesquisas envolvendo essa temática, faltam dados relativos à região Nordeste do Brasil, o que justifica a realização deste estudo, cujo objetivo é correlacionar o EN ao nível de QV de pacientes em HD.

\section{MÉTODOS}

Estudo transversal, conduzido no Hospital das Clínicas da Universidade Federal de Pernambuco, durante o período de março a julho de 2012, com pacientes adultos de ambos os sexos que fazem parte do programa de HD do referido local. Foram incluídos pacientes portadores de DRC que iniciaram o tratamento dialítico há no mínimo três meses e que tivessem idade igual ou superior a 18 anos. Foram excluídos os pacientes que apresentaram déficit cognitivo ou alteração orgânica que tornasse inviável a aplicação do questionário, gestantes, pacientes acamados e os que possuíssem antecedente pessoal de transplante renal.

Optou-se por entrevista, realizada durante as sessões de HD. Os dados demográficos, socioeconômicos e clínicos foram: idade, sexo, procedência, posse de itens, grau de instrução do chefe da família, etiologia da doença renal, tempo de manutenção e duração da sessão de HD, adequação da dose de diálise e patologias associadas. A avaliação da adequação da dose de diálise (Kt/V) foi realizada por meio da fórmula de Daugirdas II. ${ }^{5}$ Para determinar a classe socioeconômica, foi utilizado o Critério de Classificação Econômica Brasil (CCEB), estabelecido pela Associação Brasileira de Antropologia e Associação Brasileira de Empresas de Pesquisa $(A B E P)^{6}$, e posteriormente agrupados em classe econômica alta e baixa. ${ }^{7}$

Foram coletados dados de altura, índice de massa corporal (IMC), circunferência do braço (CB), prega cutânea tricipital (PCT) e circunferência mus- 
cular do braço (CMB). O peso seco, obtido logo após a sessão de HD, foi aferido em balança eletrônica da

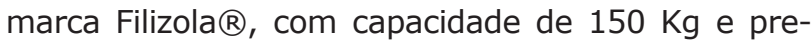
cisão de $100 \mathrm{~g}$. A altura foi obtida por meio de um estadiômetro acoplado à balança, com capacidade para $1,90 \mathrm{~m}$ e precisão de $1 \mathrm{~mm}$, conforme a técnica de Lohman et al ${ }^{8} \mathrm{O}$ IMC, razão entre o peso seco e o quadrado da altura, foi classificado de acordo com a OMS. ${ }^{9} \mathrm{~A}$ CB, em centímetros, foi obtida com o paciente em pé, no ponto médio do braço contralateral à fístula arteriovenosa funcionante (no caso de fístula arteriovenosa em ambos os braços, foi utilizado o braço direito), utilizando-se uma fita métrica inelástica. Para a PCT, em milímetros, foi utilizado um adipômetro calibrado, da marca Lange, no mesmo ponto médio marcado para a aferição da CB. A CMB foi obtida por meio da fórmula $\mathrm{CMB}(\mathrm{cm})=\mathrm{CB}(\mathrm{cm})-0,314 \times$ PCT $(\mathrm{mm})$. Os resultados da CB, PCT e CMB foram comparados aos definidos para a população normal por Frisancho ${ }^{10}$ e as classificações do estado nutricional foram obtidas por meio da porcentagem do ideal pela classificação de Blackburn \& Thornton. ${ }^{11}$

Os dados bioquímicos avaliados foram ureia pré e pós-diálise (para o cálculo do Kt/V), creatinina sérica $(\mathrm{Cr})$ e albumina sérica (Alb), utilizando valores de referência para pacientes em HD, a saber: Alb > 3,5 mg/dL e Cr entre 7-12 mg/dL. ${ }^{12}$

Para avaliar a QV foi utilizado o instrumento Medical Outcomes Study-36 - Item Short Form Health Survey (SF-36), um instrumento genérico, traduzido e validado para a língua portuguesa por Ciconelli et al. ${ }^{13}$

Os dados foram processados através do software SPSS 20.0. Todos os testes foram aplicados com $95 \%$ de confiança. As variáveis numéricas foram representadas pelas medidas de tendência central e medidas de dispersão. Todas as variáveis quantitativas foram testadas quanto à normalidade pelo teste de Kolmogorov-Smirnov. As que apresentaram distribuição normal tiveram os seus resultados expressos como média e desvio padrão e as que apresentaram distribuição não-normal e/ou não homogênea, como mediana e intervalo interquartílico (entre $25 \%$ e $75 \%$ ). Para a correlação entre as dimensões do SF-36 com os indicadores nutricionais, tempo de HD, $\mathrm{Kt} / \mathrm{V}$ e idade foram utilizados Coeficiente de Correlação de Pearson e/ou Spearman's.
A coleta de dados foi iniciada após autorização do Comitê de Ética em Pesquisa em Seres Humanos do Centro de Ciências da Saúde da Universidade Federal de Pernambuco, de acordo com a Resolução n. ${ }^{\circ}$ 196/96 do Conselho Nacional de Saúde, sob o registro de n. ${ }^{\circ} 474124$.

\section{RESULTADOS}

Dos 52 participantes do programa de HD do referido local, 37 atenderam aos critérios de inclusão no estudo. A idade média foi de $50,6 \pm 13,0$ anos, e 54,1\% $(n=20)$ da amostra pertenciam ao sexo feminino. Quanto à procedência, $91,9 \%(n=34)$ eram da área urbana e todos os pacientes pertenciam à classe econômica baixa.

As principais etiologias da DRC foram a HAS $(24,3 \% ; n=9)$, etiologia indeterminada (24,3\%; $\mathrm{n}=9)$ e o DM $(19,0 \% ; n=7)$. As demais causas foram distribuídas entre litíase renal (10,8\%; $\mathrm{n}=4)$; nefrite lúpica $(5,4 \% ; \mathrm{n}=2)$; mieloma múltiplo $(5,4 \% ; n=2)$, entre outras.

Ao avaliar o tempo de HD em meses foi encontrado que $56,8 \%(n=21)$ da amostra realizavam o procedimento há menos de um ano. O valor médio da adequação da dose de diálise foi $1,76 \pm 0,76$, com $73 \%(n=27)$ dos pacientes apresentando Kt/V > 1,2 (adequado).

A distribuição dos pacientes segundo o EN encontra-se na Tabela 1.

Quanto aos dados laboratoriais, o valor médio de Alb da amostra encontrava-se dentro dos limites de referência $(3,7 \pm 4,3 \mathrm{~g} / \mathrm{dL})$, assim como os resultados de $\mathrm{Cr}(10,1 \pm 3,5 \mathrm{mg} / \mathrm{dL})$.

Ao avaliar a QV, os aspectos físicos foram os mais comprometidos, enquanto os aspectos sociais, a saúde mental e os aspectos emocionais apresentaram menor repercussão. Os escores das dimensões do SF-36 encontram-se na Tabela 2.

$\mathrm{Na}$ análise das correlações, O IMC esteve diretamente correlacionado com os aspectos emocionais (AE) $(r=0,350)$, a CB com a vitalidade $(r=0,385)$, a Alb com a capacidade funcional (CF) ( $r=0,370$ e 0,327 , respectivamente) e o tempo de HD com os aspectos físicos $(r=0,502)$, conforme Tabela 3. 


\section{Tabela 1}

Distribuição dos pacientes do programa de hemodiálise do Hospital das Clínicas da Universidade Federal de Pernambuco segundo o estado nutricional por diferentes parâmetros.

\begin{tabular}{|c|c|c|c|}
\hline Variáveis & $\mathrm{n}$ & $\%$ & IC $95 \%$ \\
\hline \multicolumn{4}{|l|}{ Índice de Massa Corporal } \\
\hline$<18,5$ = Desnutrição & 3 & 8,1 & \\
\hline $18,5 \mid-25,0=$ Eutrofia & 18 & 48,7 & \\
\hline$\geq 25,0=$ Excesso de peso & 16 & 43,2 & \\
\hline \multicolumn{4}{|l|}{ Circunferência Braquial } \\
\hline$<90 \%=$ Desnutrição & 14 & 37,8 & \\
\hline $91-110 \%=$ Eutrofia & 15 & 40,5 & \\
\hline$>110 \%=$ Excesso de peso & 8 & 21,7 & \\
\hline \multicolumn{4}{|l|}{ Circunferência Muscular do Braço } \\
\hline$<90 \%=$ Desnutrição & 14 & 37,8 & \\
\hline$>90 \%=$ Eutrofia & 23 & 62,2 & \\
\hline \multicolumn{4}{|l|}{ Prega Cutânea Tricipital } \\
\hline$<90 \%$ = Desnutrição & 14 & 37,8 & \\
\hline $91-110 \%=$ Eutrofia & 6 & 16,3 & \\
\hline$>110=$ Excesso de peso & 17 & 45,9 & \\
\hline \multicolumn{4}{|l|}{ Albumina sérica } \\
\hline$<3,5 \mathrm{~g} / \mathrm{dl}=$ Desnutrição & 8 & 25,0 & \\
\hline$\geq 3,5 \mathrm{~g} / \mathrm{dl}=$ Eutrofia & 24 & 75,0 & \\
\hline \multicolumn{4}{|l|}{ Creatinina sérica } \\
\hline $\begin{aligned}<7 \mathrm{mg} / \mathrm{dL} & =\text { Desnutrição } \\
>7 \mathrm{mg} / \mathrm{dL} & =\text { Eutrofia }\end{aligned}$ & $\begin{array}{c}7 \\
30\end{array}$ & $\begin{array}{l}18,9 \\
81,1\end{array}$ & \\
\hline
\end{tabular}

Fonte: Produção dos autores.

\section{Tabela 2}

Escores das dimensões do questionário SF-36 dos pacientes do programa de hemodiálise do Hospital das Clínicas da Universidade Federal de Pernambucow

\begin{tabular}{|c|c|c|c|c|c|c|c|}
\hline$S F-36$ & Média & Desvio Padrão & Mediana & Q1 & Q3 & Mínimo & Máximo \\
\hline Capacidade Funcional $^{1}$ & 50,4 & 34,0 & - & - & - & 0,0 & 100,0 \\
\hline Estado Geral de Saúde ${ }^{1}$ & 53,3 & 23,8 & - & - & - & 5,0 & 92,0 \\
\hline Dor $^{1}$ & 52,5 & 34,0 & - & - & - & 0,0 & 100,0 \\
\hline Vitalidade $^{1}$ & 54,2 & 26,7 & - & - & - & 5,0 & 100,0 \\
\hline Saúde Mental ${ }^{1}$ & 74,8 & 22,2 & & & & 20,0 & 100,0 \\
\hline Aspectos Sociais ${ }^{2}$ & - & - & 100,0 & 56,3 & 100,0 & 0,0 & 100,0 \\
\hline Aspectos Emocionais² & - & - & 100,0 & 16,7 & 100,0 & 0,0 & 100,0 \\
\hline Limitação por aspectos físicos ${ }^{2}$ & - & - & 0,0 & 0,0 & 25,0 & 0,0 & 100,0 \\
\hline
\end{tabular}

Fonte: Produção dos autores.

(1) Distribuição normal.

(2) Distribuição não normal. 


\section{Tabela 3}

Correlação entre as pontuações das dimensões do SF-36 e parâmetros antropométricos, bioquímicos e clínicos dos pacientes do programa de hemodiálise do Hospital das Clínicas da Universidade Federal de Pernambuco

\begin{tabular}{lcccccccc}
\hline Variáveis & $\mathrm{CF}^{1}$ & $\mathrm{DF}^{1}$ & $\mathrm{EGS}^{1}$ & $\mathrm{Vit}^{1}$ & $\mathrm{SM}^{1}$ & $\mathrm{LAF}^{2}$ & $\mathrm{AS}^{2}$ & $\mathrm{AE}^{2}$ \\
\hline IMC & $-0,029$ & 0,035 & 0,213 & 0,293 & 0,058 & 0,205 & $-0,034$ & $0,350 *$ \\
CB & 0,116 & 0,169 & 0,188 & $0,385^{*}$ & 0,108 & 0,272 & 0,086 & 0,270 \\
CMB & 0,068 & 0,066 & 0,199 & 0,305 & 0,045 & 0,209 & 0,121 & 0,145 \\
PCT & 0,032 & 0,199 & 0,023 & 0,299 & 0,187 & 0,216 & $-0,030$ & 0,263 \\
Creatinina & 0,231 & 0,472 & 0,669 & 0,411 & 0,998 & 0,835 & 0,208 & 0,182 \\
Hemoglobina & $0,327 *$ & 0,089 & 0,010 & 0,314 & 0,085 & 0,310 & 0,033 & $-0,065$ \\
Tempo em hemodiálise ${ }^{2}$ & $-0,102$ & 0,003 & $-0,058$ & 0,137 & 0,291 & $0,502 *$ & 0,066 & 0,015 \\
Kt/V & 0,032 & $-0,075$ & 0,004 & $-0,062$ & $-0,219$ & $-0,086$ & 0,067 & 0,177 \\
Idade & $-0,302$ & $-0,298$ & 0,197 & $-0,222$ & $-0,150$ & -0244 & $-0,251$ & $-0,058$ \\
\hline
\end{tabular}

(1) Correlação de Pearson (2) Correlação de Spearman's $\left({ }^{*}\right)$ Correlação significativa.

CF (Capacidade Funcional), DF (dor física), EGS (estado geral de saúde), Vit (vitalidade), SM (saúde mental), LAF (limitação por aspectos), AS (aspectos sociais), AE (aspectos emocionais).

Fonte: Produção dos autores.

\section{DISCUSSÃO}

Quanto às características da população estudada, houve prevalência do sexo feminino, diferindo dos dados da literatura ${ }^{14}$, enquanto a idade média foi semelhante a de outros estudos nacionais. ${ }^{15,16}$ A DRC é mais comum com o avanço da idade, fase onde ocorre redução fisiológica da filtração glomerular e surgem lesões renais secundárias a doenças crônicas comuns da idade. ${ }^{17}$

Além dos idosos, indivíduos hipertensos e diabéticos são considerados grupos de risco para desenvolver a DRC. ${ }^{18}$ Neste estudo, além da etiologia indeterminada, o DM e a HAS foram as principais causas da doença, sendo esta última, a principal comorbidade apresentada pelos pacientes. O rim e a HAS possuem importante interação, visto que uma alteração na função renal muitas vezes está associada à fisiopatologia da hipertensão primária, e a DRC, por sua vez, é a causa mais comum de HAS secundária. ${ }^{19}$

A população investigada demonstrou, em sua totalidade, viver em condições socioeconômicas desfavoráveis e possuir baixo nível de instrução, o que pode dificultar a adesão e compreensão do tratamento e, consequentemente, comprometer a QV. Quanto à avaliação do EN, foi encontrada uma prevalência de 48,7\% $(n=18)$ de eutrofia considerando o IMC, semelhante a dados da literatura. ${ }^{20} \mathrm{~A}$ desnutrição, por sua vez, foi observada em $8,1 \%(n=3)$ dos pacientes e resultados semelhantes também foram observados por outros autores. ${ }^{20,21}$

Apesar do IMC ser bastante utilizado na prática clínica devido a sua praticidade, este índice possui baixa sensibilidade em diagnosticar a desnutrição em pacientes em HD devido, entre outros fatores, à retenção líquida comum nesses pacientes. ${ }^{22}$

Outros parâmetros antropométricos podem ser utilizados na avaliação da composição corporal. No presente estudo, através de CMB foi observado um percentual de eutrofia de $62,2 \%(n=23)$, corroborando com outros autores que encontraram $55,0 \%$ de eutrofia pelo mesmo parâmetro. ${ }^{21}$ A CB que classificou 40,5\% $(n=15)$ como eutróficos divergiu dos dados de Vegine et al. ${ }^{23}$, que encontraram diagnóstico de eutrofia em 100\% de sua amostra de acordo com esse parâmetro. Por outro lado, a avaliação da PCT evidenciou um percentual de eutrofia de $16,3 \%(n=6)$, corroborando com estudo de Koehnlein et al. ${ }^{22}$, que encontrou $12,0 \%$ de eutrofia pela PCT.

Portanto, o monitoramento periódico do EN deve fazer parte do seguimento de pacientes em HD, sendo fundamental para prevenir, diagnosticar e tratar a desnutrição proteico-calórica. ${ }^{21}$

A avaliação bioquímica demonstrou que $75 \%$ dos pacientes apresentaram Alb adequada ( $\geq 3,5 \mathrm{~g} / \mathrm{dL}$ ), que é o método bioquímico mais utilizado devido à facilidade de sua medição e asso- 
ciação com efeitos clínicos. Porém, ela pode ser significativamente alterada pela DRC e nem sempre refletir o estado nutricional, através de perdas pelo dialisato, doenças sistêmicas, hiper-hidratação, idade avançada e presença de inflamação. ${ }^{24}$ Infelizmente, neste estudo não foi possível analisar marcadores de inflamação, o que proporcionaria um diagnóstico mais acurado da hipoalbuminemia. Os níveis séricos reduzidos de $\mathrm{Cr}$ em 19,9\% da amostra podem refletir redução da massa muscular e/ou ingestão proteica insuficiente, associando-se a maiores taxas de mortalidade. ${ }^{25}$

Além dos indicadores nutricionais, é fundamental conhecer a eficácia do procedimento dialítico, visto que uma diálise inadequada mantém o paciente em uremia, o que leva à anorexia, proporcionando alterações desfavoráveis no estado nutricional. ${ }^{26}$ Neste estudo, $27 \%$ dos pacientes apresentaram inadequação da diálise segundo o Kt/V.

Embora possam ser utilizados diversos marcadores nutricionais na DRC, não existe um parâmetro isolado, capaz de avaliar o EN desses pacientes devido às alterações provocadas pela própria enfermidade. ${ }^{26}$ É recomendável, como foi feito nesta pesquisa, utilizar vários parâmetros em conjunto para contornar as limitações de cada método, melhorando a acurácia e a precisão do diagnóstico nutricional.

Neste estudo, também foi possível observar alterações na QV, com menor pontuação para aspectos físicos, indicando o importante impacto da saúde física no desempenho de atividades diárias e/ou profissionais. Esta variável não apresenta um consenso na literatura já que existem estudos em que pacientes em HD pareciam ter um desempenho físico melhor ${ }^{27}$, enquanto outros discordavam. ${ }^{28}$ Sabe-se que o tratamento hemodialítico é monótono e restrito, levando à deficiência funcional e ao sedentarismo, refletindo diretamente na QV.

A capacidade funcional, dimensão com a segunda menor pontuação, é avaliada a partir das atividades diárias como capacidade de cuidar de si, vestir-se, tomar banho, subir escadas. Comparando os pacientes portadores de DRC aos demais cidadãos, observa-se que eles apresentam uma capacidade funcional precária, visto que possuem diversos fatores que prejudicam a condição física como anemia, cardiopatia, HAS, neuropatia urêmica, fadiga e dores em membros inferiores. ${ }^{26}$

Por outro lado, as dimensões menos comprometidas foram aspectos sociais, aspectos emocionais e saúde mental. Os aspectos sociais relacionam-se às consequências da saúde física e mental nas atividades sociais, como interação com amigos e parentes. ${ }^{13}$ Porém, estas alterações não foram observadas neste estudo, demonstrando ser o componente que menos compromete a QV, corroborando com Silveira et al. ${ }^{28}$ Entretanto, ao comparar pacientes em HD e em diálise peritoneal, Gonçalves et al. ${ }^{15}$ confirmam que estes últimos apresentaram maiores índices quanto às atividades sociais.

Os aspectos emocionais apontam os reflexos das condições emocionais no desempenho das atividades diárias ${ }^{18}$, enquanto a dimensão saúde mental avalia presença de nervosismo, depressão, ansiedade, tranquilidade e felicidade. No presente trabalho, os pacientes se mostraram otimistas e conformados com o tratamento, sem que isto refletisse em seu dia-a-dia, corroborando com resultados de Stasiak et al. ${ }^{14}$

As variáveis que se correlacionaram com QV foram tempo em HD, IMC, CB e Alb. O tempo de $H D$, por sua vez, se correlacionou positivamente com os aspectos físicos. Porém, em estudo com 125 indivíduos da unidade de diálise do Hospital de Base de São José do Rio Preto, o componente físico mostrou correlação negativa com tempo de $H D$, indicando que quanto maior o tempo de HD, mais baixos foram os valores desse componente. ${ }^{29}$ Confirmando a maior intolerância ao tratamento pelos pacientes que dialisam há menos tempo.

Ao avaliar a correlação entre o EN e a QV, observa-se que o IMC apresentou correlação positiva com os aspectos emocionais. Santos ${ }^{30}$ encontrou que mulheres classificadas como desnutridas pelo IMC apresentaram menor pontuação para o estado geral de saúde, dimensão que pertence aos componentes físico e mental de QV.

A CB também apresentou correlação positiva com a vitalidade, dimensão que diz respeito à disposição do paciente em realizar tarefas do cotidiano. Entretanto, o estudo The Reduction of Morbidity and Mortality Hemodialysis Patientes $(\mathrm{HEMO})^{31}$, realizado com pacientes de diversas unidades de diálise norte-americana, 
não observou nenhuma associação estatisticamente significativa entre $\mathrm{CB}$ e variáveis do SF-6. É importante salientar que pesquisas que avaliem esse parâmetro antropométrico na população em tratamento dialítico são escassas.

A Alb sérica se correlacionou positivamente com a dimensão de capacidade funcional. Essa correlação é provável, pois a Alb é considerada marcador da complexa síndrome desnutrição-inflamação, além de já estar bem documentado a sua influência sobre a morbimortalidade de pacientes em HD. ${ }^{24}$

Por outro lado, sabe-se que as concentrações séricas de creatinina são indicativas de proteínas somáticas, massa muscular e estado nutricional. A perda e/ou fraqueza muscular é comum em pacientes em HD, portanto parece razoável que a capacidade funcional reduzida esteja associada com decréscimo da $\mathrm{Cr}$ sérica. Entretanto, não foi observada correlação entre os níveis de $\mathrm{Cr}$ e o nível de QV.

A CMB e a PCT, não apresentaram correlação com as dimensões do SF-36, o que pode ter ocorrido devido ao número reduzido de participantes da pesquisa.

Ao lado de medidas objetivas (laboratoriais e antropométricas) visando a detecção de distúrbios clínicos ocasionados pela DRC, preconiza-se a monitorização da QV percebida pelos pacientes mantidos em HD. ${ }^{32}$ Com a melhora da sobrevida, a busca por melhor QV tem se tornado um dos principais objetivos da terapia dialítica crônica.

Afinal, a correlação entre avaliações objetivas que abrangem fatores modificáveis, tais como estado nutricional, e avaliações subjetivas de aspectos de grande interesse, como QV, auxilia na estratégia de intervenções prioritárias pela equipe de saúde.

Algumas limitações do estudo abrangem o pequeno número amostral, o qual não permite que inferências mais adequadas pudessem ser realizadas; e a ausência de ajustes para fatores de confusão como a idade, o tempo de HD, comorbidades, anemia e adequação dialítica, que podem interferir sobre o EN e a QV.

\section{CONCLUSÃO}

Os pacientes em HD apresentaram importante comprometimento da $\mathrm{QV}$, principalmente no que diz respeito aos aspectos físicos. Além disso, indica- dores nutricionais como o IMC, CB e Alb correlacionaram-se com dimensões do SF-36, indicando que alterações no EN podem influenciar o funcionamento físico e emocional desses pacientes.

\section{REFERÊNCIAS}

1. Romão Junior JE. Doença Renal Crônica: Definição, Epidemiologia e Classificação. J Bras Nefrol. 2004;26(3 supl. 1):1-3. Disponível em: http://www.bjn.org.br/details/1183/pt-BR/doenca-renal-cronica--definicao--epidemiologia-e-classificacao

2. Sesso RC, Lopes AA, Thomé FS, Lugon JR, Martins CT. Inquérito Brasileiro de Diálise Crônica 2016. J Bras Nefrol. 2017;39(3):261-266. Disponível em: http://www. scielo.br/pdf/jbn/v39n3/pt_0101-2800-jbn-39-03-0261.pdf

3. Sánchez SH, López DG, Lozano AS, González-Calvo G, Tejerina MB, Vallejo NG. Valoración física, condición física y calidad de vida en pacientes com diferentes tratamientos renales sustitutivos. Enferm Nefrol. 2015;18(2):81-88. Disponível em: http://scielo.isciii.es/pdf/enefro/v18n2/original1.pdf

4. Guedes KD, Guedes HM. Qualidade de vida do paciente portador de insuficiência renal crônica. Revista Ciência \& Saúde. 2012;5(1):48-53. Disponível em: http://revistaseletronicas.pucrs.br/ojs/index.php/faenfi/article/ view/9734/7746

5. Daugirdas JT. Prescrição de hemodiálise crônica: uma abordagem da cinética da uréia. In: Daugirdas JT, Ing TS, editors. Manual de diálise. 3a ed. Rio de Janeiro: Medsi; 2003. cap. 9.

6. ABEP. Critério de Classificação Econômica Brasil [internet].Acesso em: 05 de maio de 2017. Disponível em: http://www.abep.org

7. Christofaro DGD, Andrade SM, Fernandes RA, Ohara D, Dias DF, Freitas Junior IF, et al. Prevalência de fatores de risco para doenças cardiovasculares entre escolares em Londrina-PR: diferença entre classes econômicas. Rev Bras Epidemiol. 2011;14(1):27-35. Disponível em: http://www.scielo.br/pdf/rbepid/v14n1/03.pdf

8. Lohman TG, Roche AF, Martorell R. Anthropometric standardization reference manual. 1a ed. Champaign, IL: Human Kinetics Books; 1988.

9. World Health Organization. Physical status: the use and interpretation of anthropometry: WHO - Technical Report Series. Geneva: World Health Organization, 1995.

10. Frisancho, AR. New norms of upper limb fat and muscle áreas for assesments of nutritional status. Am J Clin Nutr. 1981;34(11):2540-5. doi: 10.1093/ajcn/34.11.2540

11. Blackburn GL, Thornton PA. Nutritional assessment of the hospitalized patients. Med Clin North Am. 1979;63(5):11103-15.

12. Martins C. Padrões de referência para exames laboratoriais. In: Riella MC, Martins C, editors. Nutrição e o rim. $1^{\text {a }}$ ed. São Paulo: Guanabara; 2001. p.379-83. 
13. Ciconelli RM, Ferraz MB, Santos W, Meinão I, Quaresma MR. Tradução para a língua portuguesa e validação do questionário genérico de avaliação de qualidade de vida SF-36 (Brasil SF-36). Rev bras reumatol. 1999;39(3):143-50.

14. Stasiak CES, Bazan KS, Kuss RS, Schuinski AFM, Baroni G. Prevalência de ansiedade e depressão e suas comorbidades em pacientes com doença renal crônica em hemodiálise e diálise peritoneal. J Bras Nefrol. 2014;36(3):325-31. doi: 10.5935/0101-2800.20140047

15. Gonçalves FA, Dalosso IF, Borba JMC, Bucaneve J, Valerio NMP, Okamoto CT, et al. Qualidade de vida de pacientes renais crônicos em hemodiálise ou diálise peritoneal: estudo comparativo em um serviço de referência de Curitiba - PR. J Bras Nefrol. 2015;37(4):467-74. Disponível em: http://www.scielo.br/pdf/jbn/v37n4/ 0101-2800-jbn-37-04-0467.pdf

16. Freitas ATVS, Vaz IMF, Ferraz SF, Peixoto MRG, Campos MIVM. Prevalence of malnutrition and associated factors in hemodialysis patients. Rev Nutr. 2014;27(3):357-66. Disponível em: http://www.scielo. $\mathrm{br} / \mathrm{pdf} / \mathrm{rn} / \mathrm{v} 27 \mathrm{n3} / 1415-5273-\mathrm{rn}-27-03-00357 . p d f$

17. Dutra MC, Uliano EJM, Machado DFGP, Martins T, Schuelter-Trevisol F, Trevisol DJ. Avaliação da função renal em idosos: um estudo de base populacional. J Bras Nefrol. 2014;36(3):297-303. Disponível em: http://www.scielo. br/pdf/jbn/v36n3/0101-2800-jbn-36-03-0297.pdf

18. Cruz MC, Andrade $C$, Urrutia $M$, Draibe $S$, Nogueira-Martins LA, Sesso RCC. Quality of life in patients with chronic kidney disease. Clinics. 2011;66(6):991-5. doi:10.1590/S1807-59322011000600012

19. Silva Junior GB, Bentes ACSN, Daher EF, Matos SMA. Obesity and kidney disease. J Bras Nefrol. 2017;39(1):65-69. Disponível em: http://www.scielo. br/pdf/jbn/v39n1/0101-2800-jbn-39-01-0065.pdf

20. Ferraz SF, Freitas ATVS, Vaz IMF, Campos MIVAM, Peixoto MRG, Pereira ERS. Estado nutricional e ganho de peso interdialítico de pacientes com doença renal crônica em hemodiálise. J Bras Nefrol. 2015;37(3):306-314. Disponível em: http://www.scielo.br/pdf/jbn/v37n3/ 0101-2800-jbn-37-03-0306.pdf

21. Stefaneli C, Andreoti FD, Quesada KR, Detregiachi CRP. Avaliação nutricional de pacientes em hemodiálise. J Health Sci Inst. 2010;28(3):268-71. Disponível em: https:// www.unip.br/presencial/comunicacao/publicacoes/ics/ edicoes/2010/03_jul-set/V28_n3_2010_p268-271.pdf

22. Oliveira CMC, Vidal CLC, Cristino EF, Pinheiro Jr FML, Kubrusly M. Acidose metabólica e sua associação com o estado nutricional em hemodiálise. J Bras Nefrol. 2015;37(4):458-466. doi: 10.5935/0101-2800.20150073

23. Vegine PM, Fernandes ACP, Torres MRSG, Silva MIB, Avesani CM. Avaliação de métodos para identificar desnutrição energético-protéica de pacientes em hemodiálise. J Bras Nefrol. 2011;33(1): 55-61. Disponível em: http://www.scielo.br/pdf/jbn/v33n1/v33n1a08.pdf

24. Cuppari L, Kamimura MA. Avaliação Nutricional na Doença Renal Crônica: desafios na prática cllínica. J Bras Nefrol. 2009;31(supl.1):28-35. Disponível em: http:// bjn.org.br/details/1325/pt-BR/avaliacao-nutricional-na-doenca-renal-cronica--desafios-na-pratica-clinica

25. Goldwasser $\mathrm{P}$, Kaldas AI, Barth RH. Rise in serum albumin and creatinine in the first half year on hemodialysis. Kidney Int. 1999;56(6):2260-8. doi: $10.1046 / j .1523-1755.1999 .00768 . x$

26. Ekramzadeh M, Mazloom Z, Jafari P, Ayatollahi M, Sagheb MM. Major barriers responsible for malnutrition in hemodialysis patients: challenges to optimal nutrition. Nephro urol Mon. 2014;6(6):e23158. doi: 10.5812 /numonthly. 23158

27. Arenas VG, Barros LFNM, Lemos FB, Martins MA, David-Neto E. Qualidade de vida: comparação entre diálise peritoneal automatizada e hemodiálise. Acta Paul Enferm. 2009;22:535-9. Disponível em: http://www.scielo.br/pdf/ape/v22nspe1/17.pdf

28. Silveira CB, Pantoja IKOR, Silva ARM, Azevedo RN, Sá NB, Turiel MGP, et al. Qualidade de vida em pacientes em hemodiálise em um hospital público de Belém - Pará. J Bras Nefrol. 2010; 32(1):39-44. Disponível em: http://www.scielo.br/pdf/jbn/v32n1/v32n1a08.pdf

29. Martins MRI, Cesarino CB. Qualidade de vida de pessoas com doença renal crônica em tratamento hemodialítico. Rev Lat Am Enfermagem. 2005;13(5):670-6. Disponível em: http://www.scielo.br/pdf/rlae/v13n5/v13n5a10.pdf

30. Santos PR. Relação do sexo e da idade com nível de qualidade de vida em renais crônicos hemodialisados. Rev Assoc Med Bras. 2006;52(5):356-9. Disponível em: http://www.scielo.br/pdf/ramb/v52n5/a26v52n5.pdf

31. Allen $\mathrm{KL}$, Miskulin $\mathrm{D}$, Yan $\mathrm{G}$, Dwyer JT, Frydrych $\mathrm{A}$, Leung J, et al. Association of Nutritional Markers With Physical and Mental Health Status in Prevalent Hemodialysis Patients From the HEMO Study. J Ren Nut. 2002;12(3):160-9. doi: 10.1053/jren.2002.33512

32. Merkus MP, Jager KJ, Dekker FW, de Haan RJ, Boeschoten EW, Krediet RT. Predictors of poor outcome in chronic dialysis patients: The Netherlands Cooperative Study on the Adequacy of Dialysis. Am J Kidney Dis. 2000; 35(1):69-79. 